\title{
Li-ion Batteries Parameter Estimation Using Converter Excitation and Fusion Methods
}

\author{
Irene Peláez, Pablo García, Geber Villa and Sara Saheed \\ Dept.of Electrical, Electronics, Systems \& Computers Engineering \\ University of Oviedo, LEMUR Group \\ Gijón, 33204, Spain \\ Email: pelaezirene@uniovi.es,garciafpablo@uniovi.es,villageber@uniovi.es,saeedsarah@uniovi.es
}

\begin{abstract}
This paper proposes a fusion method to estimate the low frequency impedance of Lithium-Ion batteries by using excitation signals. The proposed excitation is delivered by the converter used for the interface of the battery. The fusion method is conducted in two steps. Firstly, the impedance is estimated by using a frequency method known as vector fitting. Despite its high accuracy, it has a moderate computational burden that makes it difficult to use in real-time application running on the embedded systems often used for battery and converter control. For that reason, in a second stage, recursive least squares algorithm will be used for the parameter estimation in real-time. The discussion includes not only the methods description and testing, but also the effects of the data used for the estimation (terminal current and voltage) as well as the discretization method used for the digital implementation. The proposal is validated by simulation and with preliminary experimental results.
\end{abstract}

\section{INTRODUCTION}

Thank to the economy of scale, Lithium-Ion batteries have become a competitive technology in the market while their price keep decreasing [1]. One of the critical parts of the battery that has a clear impact on its performance is the inner impedance. Power management, state of charge (SoC), state of health $(\mathrm{SoH})$ are all affected by this impedance [1]. In most applications, the battery cells are connected in series or parallel, forming the battery pack which is plugged to a converter. The ultimate goal is to estimate the resulting impedance using converter-side electrical variables (current and voltage at the battery terminals).

Different excitation inputs have been used for estimating the impedance in the literature [2]. DC current pulse injection is the technique most frequently used for measuring the impedance and evaluate the degradation. Furthermore, it is the only one accepted by the current standards for determining the power capability in Lithium-Ion batteries [3], [4]. The obtained impedance is also sensitive to the amplitude and pulse width [4] and its frequency resolution [5]. The discussion presented

The present work has been partially supported by the predoctoral grant programs Severo Ochoa and FPU for the formation in research and university teaching of Principado de Asturias PCTI-FICYT under the grant ID BP16133 and Spain MECD under the grant ID FPU16/06829. This work also was supported in part by the Research, Technological Development and Innovation Program Oriented to the Society Challenges of the Spanish Ministry of Economy and Competitiveness under grant MCIU-18-RTC-2017-6338-3, ENE2016-77919-R and by the European Union through ERFD Structural Funds (FEDER). in this paper evaluates how both properties impact on the final results.

The inner parameters depend on battery operation condition and they change with aging, triggering the need for estimating them online. Regarding online estimation techniques, different adaptive filtering methods have been successfully implemented in the modeling of unknown systems [6]. A complete review can be found in [7], [8]. Kalman filter is widely used in state estimation, however it has a complex computation and becomes quite difficult to be tuned when parameters and states have to be estimated simultaneously [9]. Different fusion techniques have been proposed for solving the problem of estimating both parameters and states at the same time [9]. This paper presents a novel fusion method which initializes the parameters with a frequency method known as vector fitting (VF), and uses the recursive least squares (RLS) algorithm for both estimating the states and the parameters in real time. Furthermore, a more accurate model which consists of two RC branches is considered, rather than the single RC model often used [10], [11].

VF approximates, first introduced in [12], estimates the frequency domain response of a transfer function with a fast and a robust performance. Even if very robust and accurate, the method has an important computational cost. For that reason, an RLS approach is used for the real time estimation [8]. It has to be remarked that the proposed method also considers the effect of the bidirectional dc/dc power converter needed for the battery interface, including the effect of the used inductor filter.

This paper is organized as follows. In Section II the model proposed for the battery impedance and the discrete transformation are introduced. In Section III, the parameter estimation methods and the effects of the input signal parameters, pulse width and frequency, are analyzed. In Section IV, the simulation and experimental results are exposed. To summarize, the conclusions obtained are gathered at the end of the paper.

\section{SYSTEM MODELING}

The chemical reactions that takes place in Lithium-Ion batteries can be modeled by the equivalent circuit shown in Fig. 1 [13]. $R_{s}$ represents the cabling connections and the inner resistance of the battery, which performs the electrochemical 


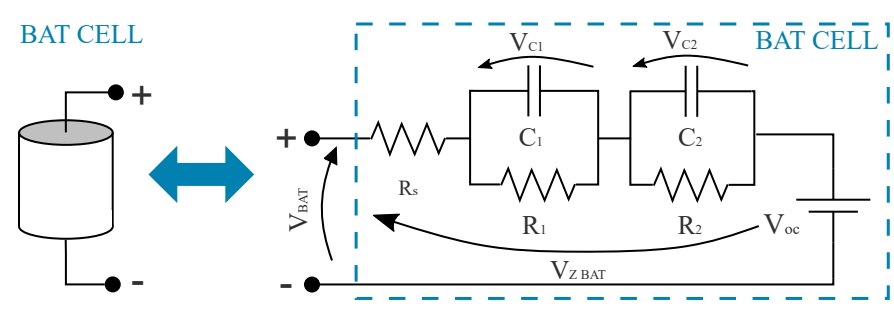

Fig. 1. Battery cell equivalent circuit.

TABLE I

RELATIONSHIP FROM CONTINUOUS TO DISCRETE TRANSFER FUNCTION SYSTEM

\begin{tabular}{|c|c|c|c|c|}
\hline & $\mathrm{ZOH}$ & Bilineal & Forwar & d Backward \\
\hline$A_{d}$ & $e^{A T_{s}}$ & $\begin{aligned} &\left(I+\frac{A T_{s}}{2}\right) . \\
& \cdot\left(I-\frac{A T s}{2}\right)^{-1}\end{aligned}$ & $\begin{array}{l}(I+ \\
\left.A T_{s}\right)\end{array}$ & $\left(I-A T_{s}\right)^{-1}$ \\
\hline$B_{d}$ & $\int_{0}^{T_{s}} e^{A \lambda} d \lambda B$ & $\left(I-\frac{A T s}{2}\right)^{-1}$ & $B T_{s}$ & $\begin{array}{c}B T_{s} . \\
\cdot\left(I-A T_{s}\right)^{-1}\end{array}$ \\
\hline$C_{d}$ & C & $\begin{array}{c}C \sqrt{T_{s}} . \\
\cdot\left(I-\frac{A T s}{2}\right)^{-1}\end{array}$ & C & $C(I-A T s)^{-1}$ \\
\hline$D_{d}$ & $D$ & $\begin{array}{c}C\left(I-\frac{A T s}{2}\right)^{-1} \\
\quad \cdot \frac{B T_{s}}{2}+D\end{array}$ & $D$ & $\begin{array}{c}C(I-A T s)^{-1} \\
\cdot B T_{s}+D\end{array}$ \\
\hline
\end{tabular}

reaction and it grows with aging [14]. The capacitive effects located in the surface of the electrode are illustrated by the first RC branch, whereas the second RC branch represents the diffusion processes of the electrolyte [14]. The continuous domain transfer function and state space representation for the impedance are gathered in (1)-(3).

$$
\begin{gathered}
z_{B A T}(s)=\frac{\frac{1}{C_{1}}}{s-\frac{1}{R_{1} C_{1}}}+\frac{\frac{1}{C_{2}}}{s-\frac{1}{R_{2} C_{2}}}+R_{s} \\
\frac{d V_{C}(t)}{d t}=A V_{C}(t)+B I_{B A T}(t) \\
V_{Z_{B A T}}(t)=C V_{C}(t)+D I_{B A T}(t) \\
A=\left|\begin{array}{cc}
\frac{-1}{R_{1} C_{1}} & 0 \\
0 & \frac{-1}{R_{2} C_{2}}
\end{array}\right| \quad B=\mid \begin{array}{cc}
\frac{1}{C_{1}} & C=\mid 1 \\
\frac{1}{C_{2}} & D=R_{s} \\
D &
\end{array}
\end{gathered}
$$

In order to conduct the estimation in a digital system, a discrete approximation for the continuous-domain representation is needed. Alternatives for the discretization are shown in Table I, which allows to perform the (2) $\rightarrow$ (4) transformation [15]. For this work, the backward transformation is used as a compromise between precision, stability [15] and computational costs.

$$
\begin{aligned}
& V_{C}[k+1]=A_{d} V_{C}[k]+B_{d} I_{B A T}[k] \\
& V_{z_{B A T}}[k]=C_{d} V_{C}[k]+D_{d} I_{B A T}[k]
\end{aligned}
$$

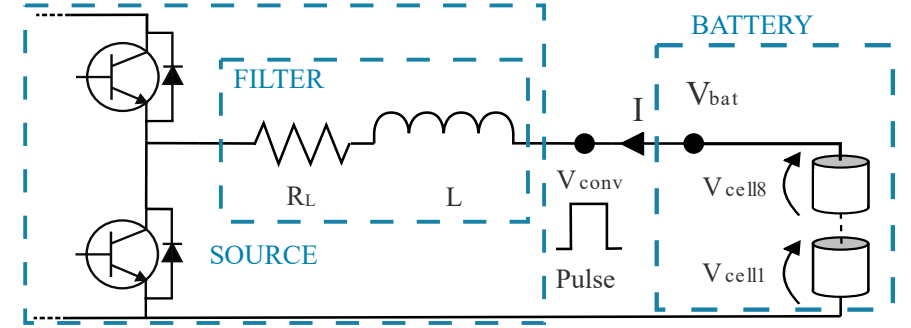

Fig. 2. Scheme for the connection of the converter to the battery terminals.

TABLE II

LITHIUM-ION CELL PARAMETERS

\begin{tabular}{ccccccc}
\hline$R_{s}[\mathrm{~m} \Omega]$ & $R_{1}[\mathrm{~m} \Omega]$ & $C_{1}[\mathrm{~F}]$ & $\tau_{1}[\mathrm{~s}]$ & $R_{2}[\mathrm{~m} \Omega]$ & $C_{2}[\mathrm{~F}]$ & $\tau_{2}[\mathrm{~s}]$ \\
\hline 24 & 0.06 & 5630 & 0.33 & 8.2 & 54277 & 445 \\
\hline
\end{tabular}

\section{Parameter estimation}

\section{A. Converter Implementation}

This work is intended for batteries which are interfaced by a converter capable of controlling the battery current by means of a current regulation. From the existing converter alternatives, a bidirectional dc/dc synchronous boost converter is considered. This converter is made up of two complementary switches series connected to which common point the output filter is connected. From the converter side, current pulses can be generated to stimulate the battery impedance, as shown in Fig. 2. The excitation signal has to be sensitive to the response of the impedance, thus the parameters can be identified in the frequency and time domain. As a first approach, the parameters obtained for a Lithium-Ion battery following the same equivalent circuit model [16] collected in Table II are used to evaluate the estimation performance by simulation.

\section{B. Methods}

1) Vector fitting: VF method seeks to obtain all the parameters from the frequency spectrum of the impedance response, which is obtained from the battery voltage and current. From the impedance frequency spectrum, the method will find the coefficients of the transfer function in (5), with closest frequency spectrum to the impedance one, as formulated in [17]-[19], where $s$ is the Laplace variable and $a_{n}$ the system poles. According to (1), the impedance consists of two poles and the $E$ component in (5) is 0 , resulting in the transfer function in (6). The coefficients in (6) are related with the battery equivalent circuit parameters as stated in (7-11).

$$
\begin{gathered}
f(s) \simeq \sum_{n=1}^{N} \frac{c_{n}}{s-a_{n}}+D+s E \\
f(s)=C \cdot(s I-A)^{-1} \cdot B+D+s E \\
=\frac{b_{2} s^{2}+b_{1} s+b_{0}}{\left(s+a_{1}\right)\left(s+a_{2}\right)}+D
\end{gathered}
$$




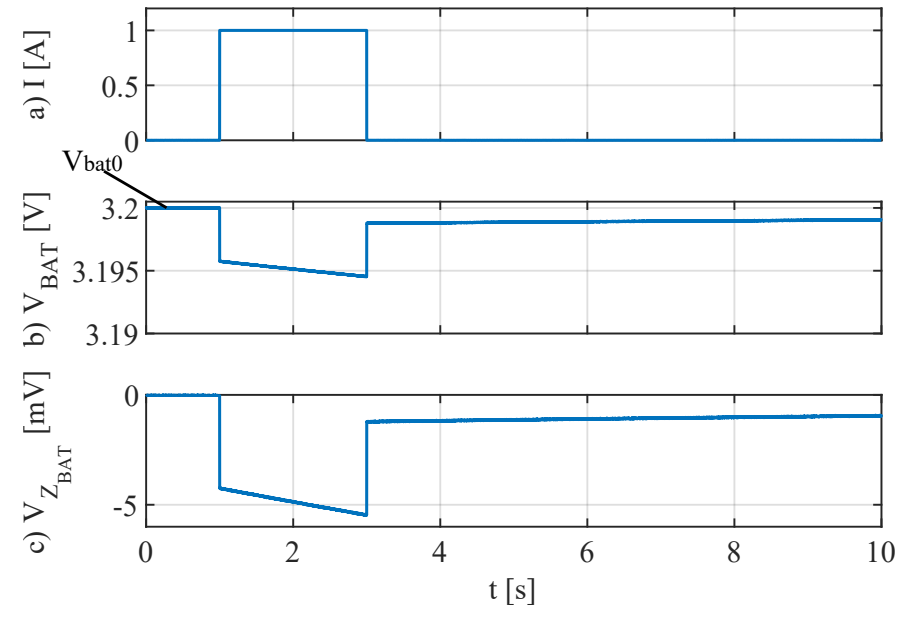

Fig. 3. a) Current pulse. b) Total battery voltage. c) Impedance battery voltage

$$
\begin{gathered}
R_{s}=D \quad \text { (7) } \quad R_{1}=b_{0}-R_{2} \quad \text { (8) } \quad C_{1}=-\frac{1}{R_{1} a_{1}} \\
R_{2}=\frac{b_{1}+\frac{1}{a_{2}} b_{0}}{\frac{-1}{a_{1}}+\frac{1}{a_{2}}} \quad \text { (10) } \quad C_{2}=-\frac{1}{R_{2} a_{2}}
\end{gathered}
$$

The VF method obtains the impedance by getting the frequency response of the current and battery voltage $\left(V_{B A T}\right)$. The digital implementation could relay on any implementation of the discrete Fourier transform (DFT). For the estimation, the DC voltage component $V_{B A T 0}$ is removed before the excitation signal starts, in order to isolate the system response to the applied impulse, as depicted in Fig. 3.

This method is digitally implemented using the following procedure: 1) a number of samples for the voltage and the current are captured and saved in two buffers, 2) the frequency domain transfer function response is obtained by calculating the DFT for both signals and using the expression (12), and 3) the VF estimation is launched to obtain the system parameters. In order to obtain a crisp resolution in the frequency domain, the different natural frequencies for the system dynamics have to be captured. According to the definition of frequency resolution in discrete systems, $f_{\text {res }}=\frac{1}{N T_{s}}$, where $N$ is the number of samples and $T_{s}$ the sample time, this resolution should be low enough to identify the large time constants of the two RC branches. The following section will present a discussion for the constrains of $f_{\text {res }}$.

$$
Z[f]=\frac{V_{Z_{B A T}}[f]}{I[f]}
$$

2) Recursive Least squares: The RLS algorithm is an adaptive filter algorithm which combines the least square estimation method plus the matrix inversion lemma [20]. As depicted in Fig. 4, this algorithm seeks to minimize a cost function, which in this case is (13), by recursively finding the coefficients of the system (3) using a forgetting factor $\lambda$ and a weighted covariance matrix $P$. In this case, not only the coefficients (3) but also the states, have to be estimated since

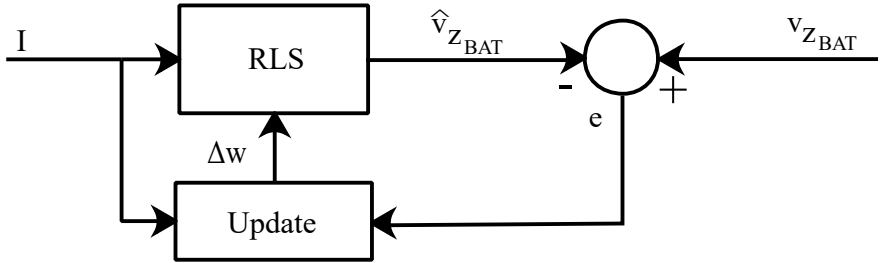

Fig. 4. Adaptive algorithm RLS scheme.

they are not observable. The final system equation is expressed as (15). There will be added further considerations due to the unobservable states before launching the RLS algorithm which are presented down below. The method is gathered in Algorithm 1, where the parameters are solved as in (16-18) following the Backward discrete transformation.

$$
\begin{gathered}
|e|=\left|\begin{array}{l}
V_{C}[k+1]-A_{d} V_{C}[k]+B_{d} I_{B A T}[k] \\
V_{z_{B A T}}[k]-C_{d} V_{C}[k]+D_{d} I_{B A T}[k]
\end{array}\right| \\
w=\left|\begin{array}{ll}
A_{d} & B_{d} \\
C_{d} & D_{d}
\end{array}\right|=\left|\begin{array}{lll}
w_{11} & w_{12} & w_{13} \\
w_{21} & w_{22} & w_{23} \\
w_{31} & w_{32} & w_{33}
\end{array}\right| \\
\left|\begin{array}{c}
V_{c 1}[k+1] \\
V_{c 2}[k+1] \\
V_{z_{B A T}}[k]
\end{array}\right|=\left|\begin{array}{lll}
w_{11} & w_{12} & w_{13} \\
w_{21} & w_{22} & w_{23} \\
w_{31} & w_{32} & w_{33}
\end{array}\right|\left|\begin{array}{c}
V_{c 1}[k] \\
V_{c 2}[k] \\
I_{i n}[k]
\end{array}\right| \\
R_{1}=\frac{-w_{13}}{w_{11}-1}, R_{2}=\frac{-w_{23}}{w_{22}-1} \\
C_{1}=\frac{T_{s} w_{11}}{w_{13}}, C_{2}=\frac{T_{s} w_{22}}{w_{23}} \\
R_{s}=w_{33}-w_{23}-w_{13}
\end{gathered}
$$

As shown in (13)-(18), the RLS method needs to estimate five parameters and two unobservable states only using two measured variables (battery terminal voltage and current). In order to reduce the order or the problem, a novel approach using a multi-resolution technique is applied. The system is a second order system, which can be split into two equivalent two first order system in series, with two different time constants for their corresponding poles. Fig. 5 represents the response of a current pulse train and the total voltage of the impedance $\left(V_{z_{B A T}}\right)$ and the hidden capacitance voltages $\left(V_{C 1}\right.$, $\left.V_{C 2}\right)$. As it can be seen, the first branch voltage maintains the value within a range, whereas the second branch has a larger time constant with an increasing trend for positive values of the average battery current. Notice that when there is no current, the second branch barely varies their voltage for this time interval, so it will be assumed to be constant.

Following the previous assumptions, the real-time estimation is conducted by using the three main steps represented in Fig. 6. Firstly, the parameters are initialized with the ones obtained in the VF method and the series resistance 


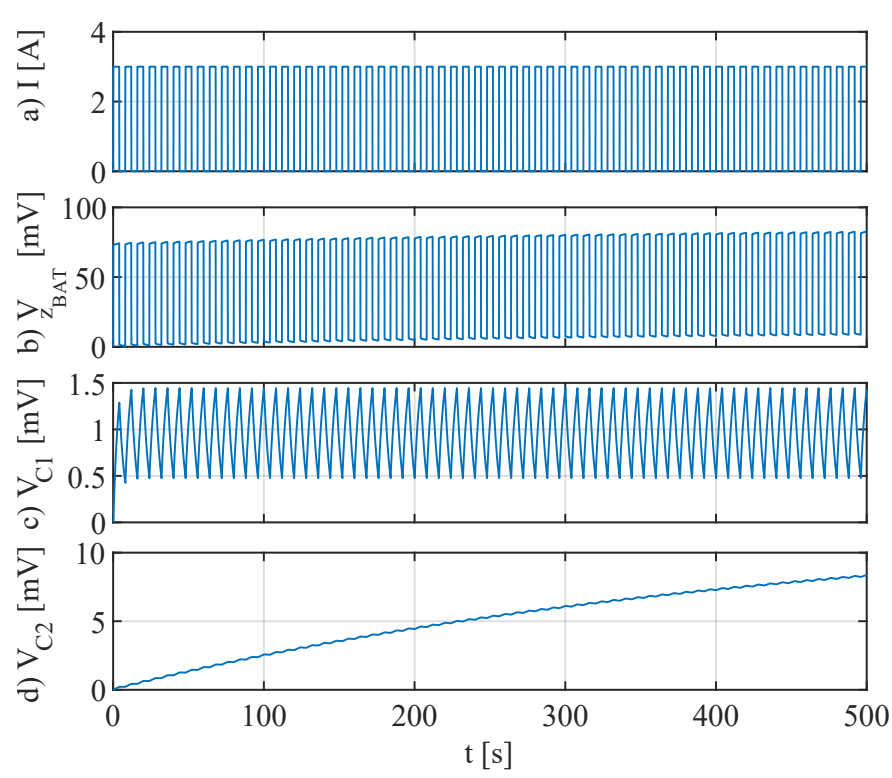

Fig. 5. Battery current and voltages: a) Current (measured) b) Voltage (measured) c) $V_{C 1}$ (unobservable) d) $V_{C 2}$ (unobservable).

is estimated every time a falling $\left(I_{F E}\right)$ or rising edge $\left(I_{R E}\right)$ in the current occurs (line 8 of Algorithm 1). The branches estimation is held during one cycle in order to determine an approximate value of the minimum voltage of the first branch $\hat{V}_{C 1 \text { min }}$, as depicted in Fig. 6 . This is the key point to decouple both voltage branches. Every time an $I_{R E}$ occurs, the estimated $\hat{V}_{C 1}$ voltage is compared to $\hat{V}_{C 1 \mathrm{~min}}$ and if it is out of a $20 \%$ range of its value, is back initialized with $\hat{V}_{C 1 \min }$ (line 11).

Finally, the second branch voltage, $\hat{V}_{C 2}$, is estimated considering two main assumptions: 1) In the interval $\left[I_{R E}[k]-\right.$ $I_{F E}[k]$, the slope of $V_{C 2}\left(\Delta \hat{V}_{C 2}\right)$ is considered to be constant and almost equal to the one during the previous interval, $\left[I_{R E}[k-1]-I_{F E}[k-1]\right.$, and 2) Between $\left[I_{F E}[k]-I_{R E}[k]\right.$, the value of $V_{C 2}$ remains constant. The step 3 is described as follows: At $I_{R E}, \hat{V}_{C 2}$ is initialized by using (19). From this point, the slope between the actual rising edge, $I_{R E}[k]$, and the previous one, $I_{R E}[k-1]$, can be determined. Assuming the $\hat{V}_{C 2}$ evolves with the slope of the previous pulse, the $\hat{V}_{C 2}$ voltage can be determined for the current pulse (line 16). As explained before, when the current vanishes to zero, $\hat{V}_{C 2}$ remains constant. Fig. 6 represents the explained decouple voltage stages, where step 1 is repeated every $I_{F E}$ and $I_{R E}$ and step 2 and 3 update the variables every $I_{R E}$ occurs.

$$
\hat{V}_{C 2 k T_{w}}=V_{z_{B A T}}-\hat{V}_{C 1}-I \hat{R}_{s k}
$$

\section{Input signal}

1) Time domain: For the RLS implementation, the shape of the input signal current has to be selected so the output voltage is sensible to the parameters sought to be estimated. For determining the influence of the input signal, a simulation is conducted in which all the states are made observable, so

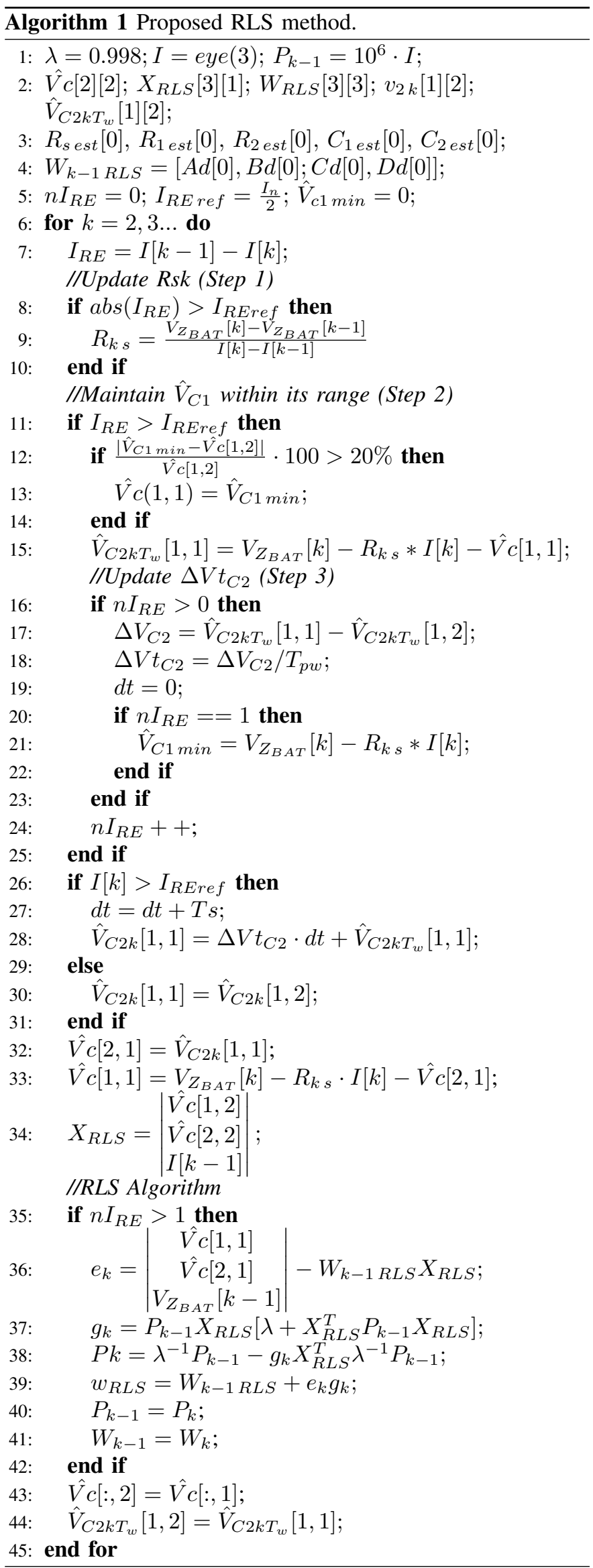



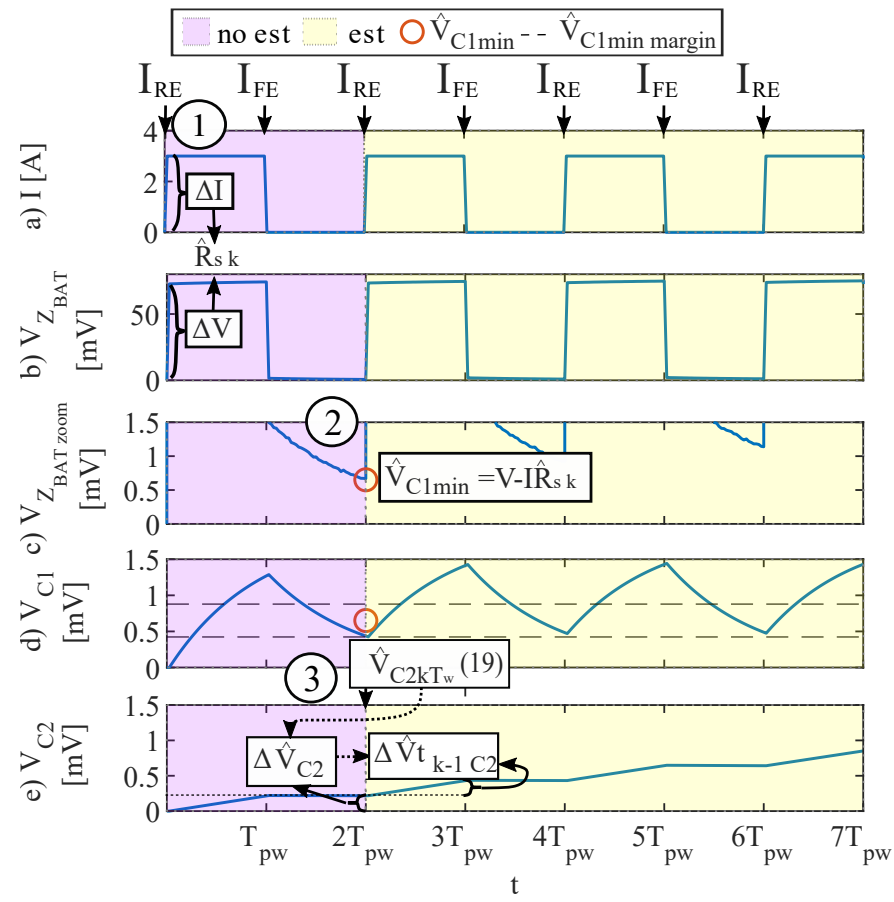

Fig. 6. Graphical explanation for the proposed multi-resolution RLS method.
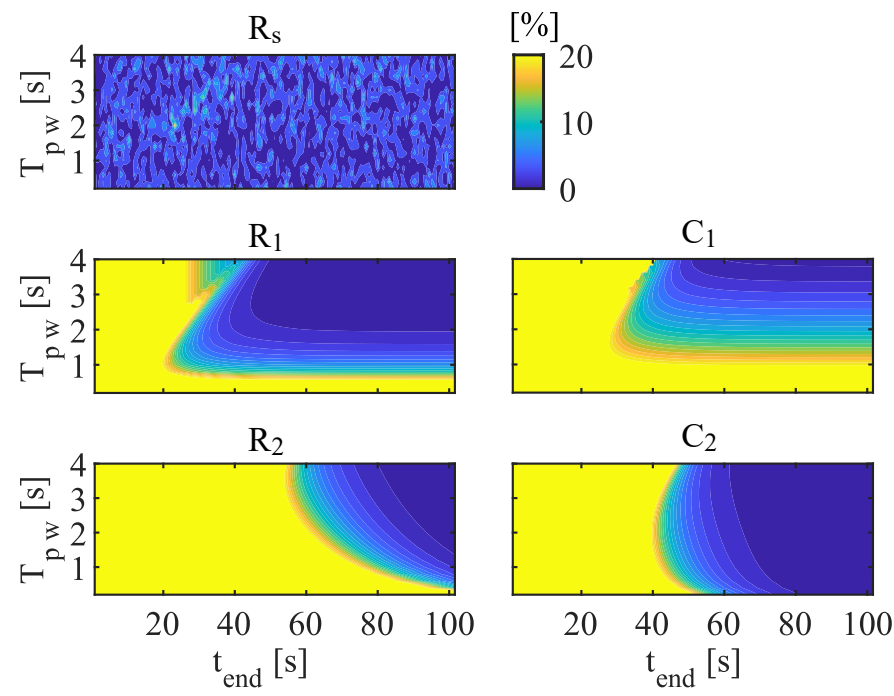

Fig. 7. Estimation error in terms of pulse width $\left(T_{p w}\right)$ for the RLS method.

the obtained error is only due to the signal itself. The width of the pulse $\left(T_{p w}\right)$ and the simulation length $\left(t_{e n d}\right)$ are varied. Each parameter error is represented in Fig. 7. The second RC branch has a larger time constant $\tau$ and it requires a longer time to obtain a the voltage evolution to identify it. Clearly, a higher $T_{p w}$ and $t_{e n d}$ results in a better estimation for both $\mathrm{RC}$ branches.

2) Frequency domain: The requirement for this domain, in which the VF method is implemented, are slightly different regarding the excitation signal. In the literature, three main input signals are commonly used: chirp [2], train of pulses
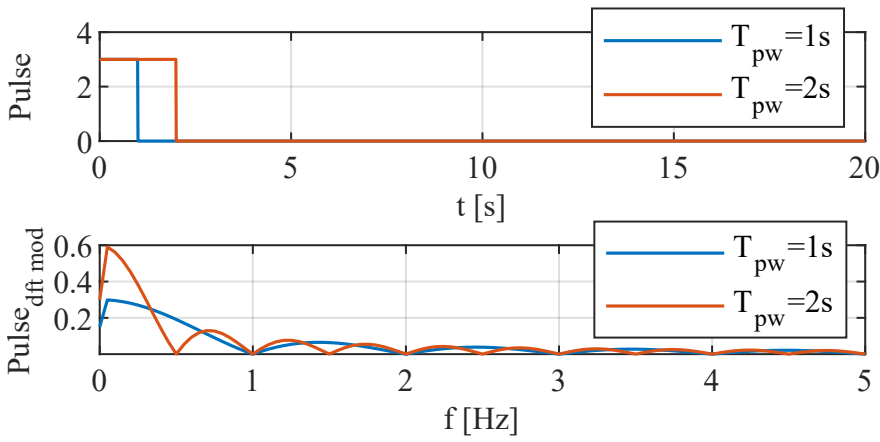

Fig. 8. Pulse in time and frequency domain for two different $T_{p w}$.

[5] or a single pulse [4]. The chirp signal has a constant magnitude over the frequency range it covers. The train of pulses will only cover the odd harmonics starting from the main frequency of the signal. This paper is focused on the low frequency range, thus, the pulse response will be enough for obtaining the response at that range. In order to examine only the impedance, the initial voltage $V_{B A T 0}$ is subtracted as it has been previously mentioned.

Fig. 8 shows the module of the DFT response of two pulse signals with same amplitude and a $T_{p w}$ of $1 \mathrm{~s}$ and $2 \mathrm{~s}$ respectively. This module vanishes to zero at $1 / T_{p w}$ and its harmonics, and also, the zero-frecuency value is proportional to $T_{p w}$ : It is doubled when $T_{p w}$ has twice the width. Since the impedance estimation requires to divide the frequency domain values of the voltage by the current, frequencies at zero-crossing points have to be removed from the estimation. Estimation results for different $f_{\text {res }}$ and pulse widths are presented in Fig. 9, where it can be observed that a lower $T_{p w}$ and $f_{r e s}$ values enhance the results. $T_{p w}$ up to $3 \mathrm{~s}$ and $f_{\text {res }}=0.7 \mathrm{mHz}$ give good results, corresponding with three times the second RC time constant.

\section{Simulation AND EXPERIMENTAL RESUlTS}

\section{A. Simulation results}

The simulation results are presented herein. Both methods will be evaluated considering the parameters of Table II.

1) VF results: Firstly, the simulated cell battery response is obtained. The VF method is evaluated by analyzing the response after a current pulse with a $T_{p w}$ of $1 \mathrm{~s}$ and a $f_{\text {res }}$ of $0.4 \mathrm{mHz}$. In Fig. 10a and 10b, the module of the frequency response for the current and the voltage are shown and from their division, the real impedance response is calculated. The real and estimated impedance is gathered in Fig. 10a and the results are collected in Table III.

2) RLS results: From the signal processed by the VF, the initial parameters for the RLS implementation are obtained. The parameters are listed in Table II. A sampling frequency of $10 \mathrm{~Hz}$ and $T_{p w}=4 \mathrm{~s}$ have been selected. The forgetting factor $\lambda$ has been set to 0.999 for the estimation and the initial covariance $P$ to $10^{6}$ by the identity matrix of (13). The parameter estimation has been achieved within $100 \mathrm{~s}$, as 

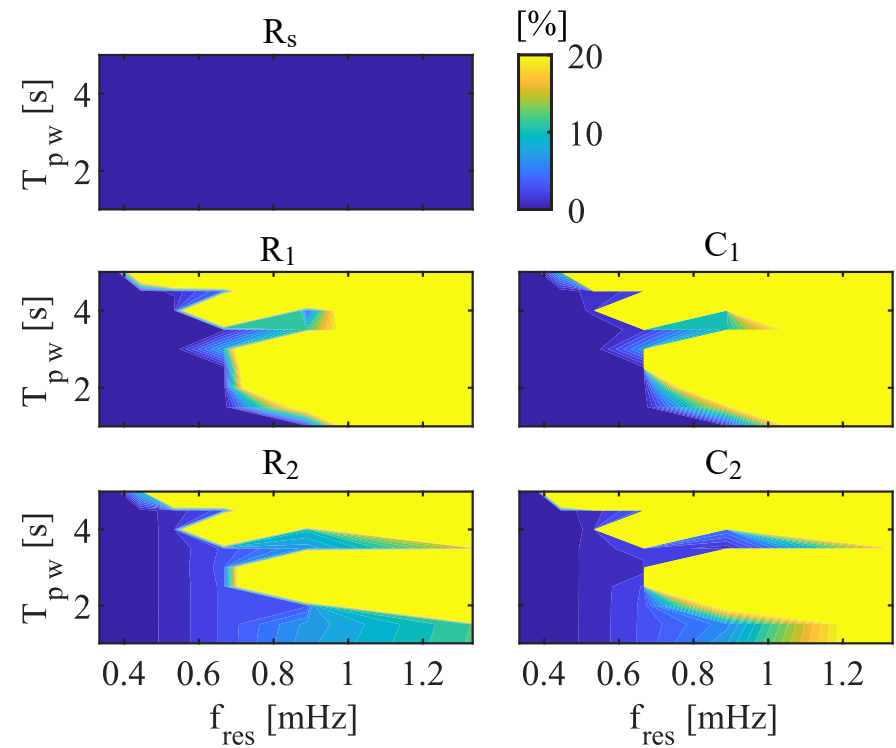

Fig. 9. Estimation error in terms of pulse width $\left(T_{p w}\right)$ for the VF method.

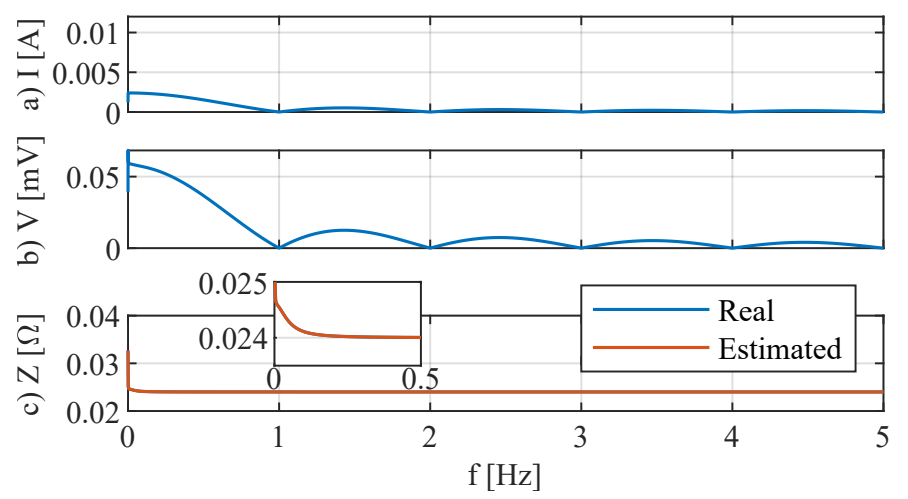

Fig. 10. Simulation results. Magnitude of the frequency response of the a) battery current, b) cell battery terminal voltage and c) cell impedance calculation using the VF method.

it can be observed in Fig. 11, which is less than four times the second RC time response. Fig. 12 presents the theoretical responses compared to the ones with the estimated parameters. The mean values of the estimation results of the last $100 \mathrm{~s}$ are gathered in Table III. The results are in good agreement for the first branch whereas they show noticeable deviations for the value of the capacitance of the second branch due to the inexact decoupling of both branch voltages and difference between $V_{C 2}$ and $\hat{V}_{C 2}$. Despite this inaccuracies, it can be seen

TABLE III

SIMULATION RESULTS

\begin{tabular}{lccccc}
\hline & $R_{s}[\mathrm{~m} \Omega]$ & $R_{1}[\mathrm{~m} \Omega]$ & $C_{1}[\mathrm{~F}]$ & $R_{2}[\mathrm{~m} \Omega]$ & $C_{2}[\mathrm{~F}]$ \\
\hline VF & 24.2 & 0.64 & 5626 & 8.1 & 55018 \\
error[\%] & 0.004 & 0.14 & 0.07 & 1.37 & 1.36 \\
\hline$R L S$ & 24.2 & 0.71 & 5432 & 9.8 & 72237 \\
error[\%] & 0.1 & 11 & 3.5 & 19 & 33 \\
\hline
\end{tabular}
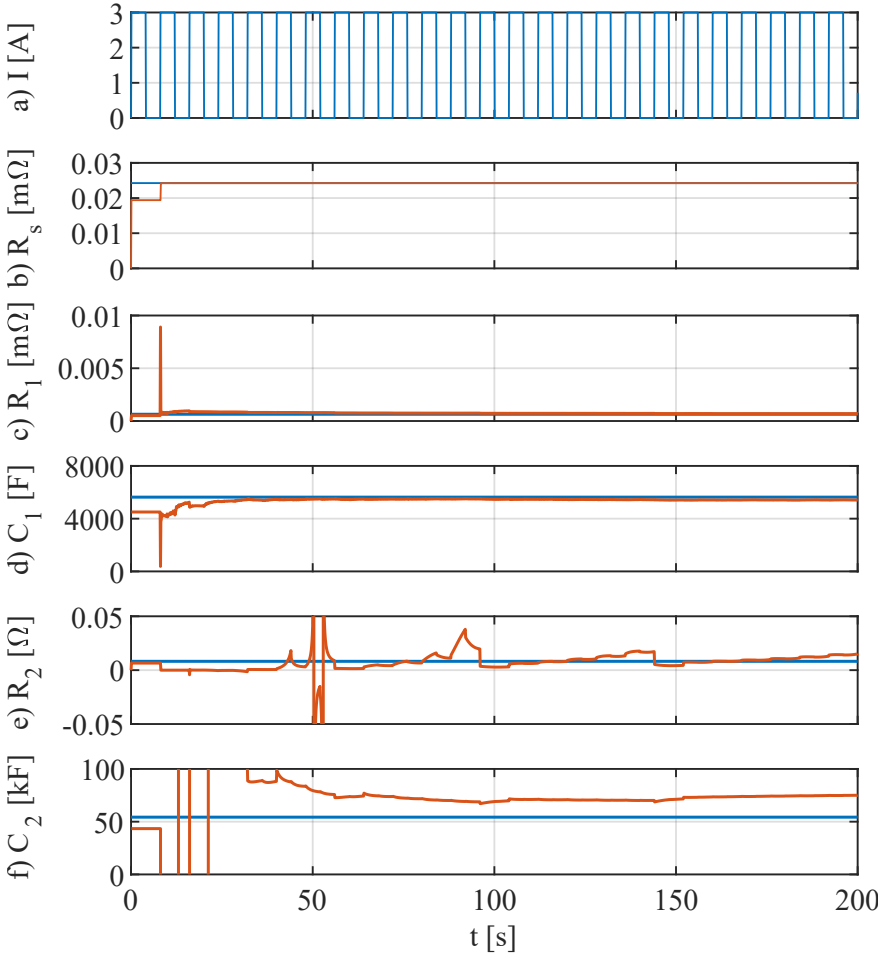

Fig. 11. Simulation results for the parameter estimation using the RLS method. Theoretical results are depicted in blue and estimated results are presented in red.
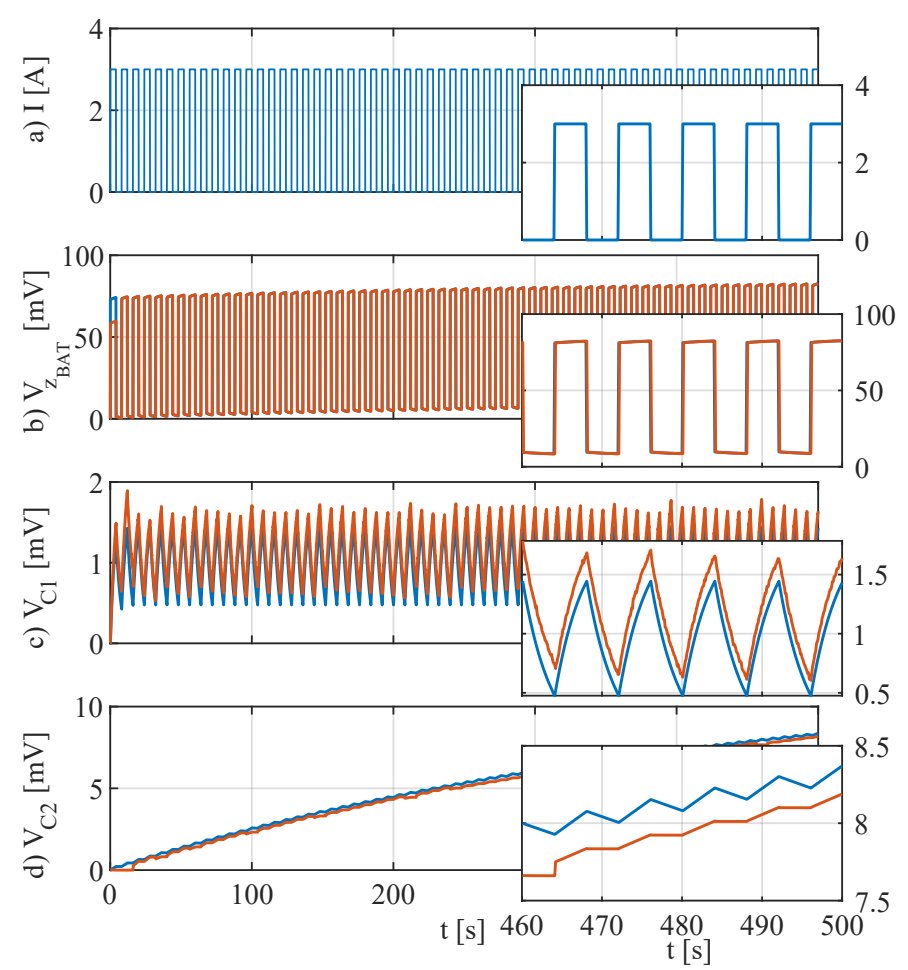

Fig. 12. Simulation results of the responses with the estimated values using the proposed multi-resolution method in red and the theoretical ones in blue.

in Fig. 12d that the estimated voltage tracks the real one. 


\section{B. Experimental results}

The experimental setup is shown in Fig. 13. The cell type used in the setup is a 26650 LPF cell with a capacity of $3.2 \mathrm{Ah}$ and nominal voltage of $3.2 \mathrm{~V}$. The battery pack is built by series connection of 8 cells. The battery terminals are connected to a bidirectional source Regatron TC.ACS.30.528.4WR.S.LC that controls the output voltage with an accuracy of $0.1 \mathrm{mV}$. The inner filter is unknown. The battery can be connected/disconnected to the source manually by a remote switch.

The data required for the estimation is acquired with a data acquisition system from National Instruments, NI 9205. These modules are capable to reach a resolution up to $0.2 \mathrm{mV}$. The cells are connected to the measurement modules by means of a non-isolated probes for the cell voltage, a $600 \mathrm{~V}$ CAT III current probe and a LEM voltage sensor LV-25 for measuring the overall battery pack voltage.

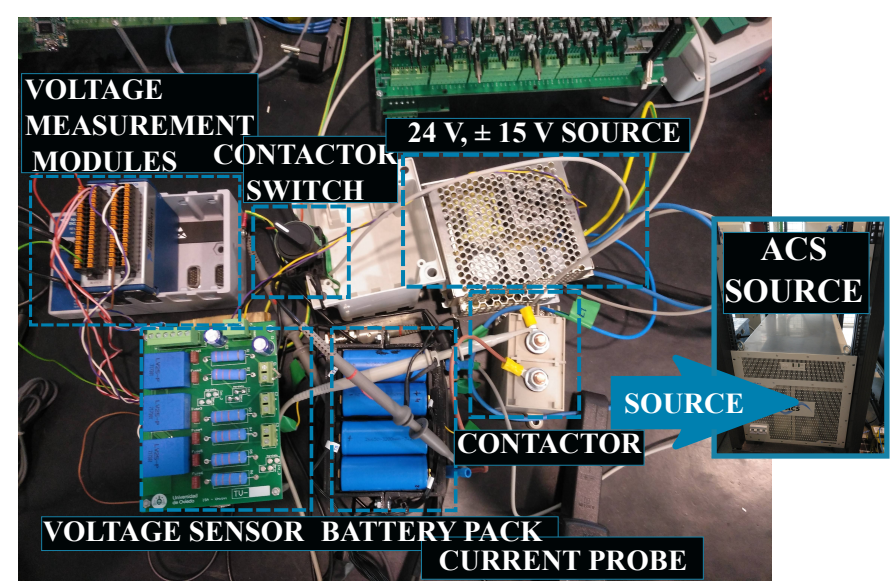

Fig. 13. Setup used for the experimental results.

1) VF estimation: Following the same steps than for the simulation results, first the frequency response of the impedance is obtained. For this purpose, a $2 \mathrm{~s}$ voltage pulse with a magnitude equal to $-3 \mathrm{~V}$ with respect to the battery pack voltage is injected and right after forced to zero, so the natural response of the battery can be obtained. The test last $45 \mathrm{~min}$ with a sampling time of $90 \mathrm{kHz}$. The high sample rate is required to avoid aliasing due to the switching harmonics delivered by the power converter. A noticeable reduction can be achieved if antialiasing filters are used for an online implementation. For a reduction of the number of samples, a pre-processing digital signal stage composed by a digital lowpass filter using a second order butterworth filter with a cut-off frequency of $100 \mathrm{~Hz}$ and a subsequent downsampling down to $2 \mathrm{kHz}$ is applied. After the preprocessing is finished, a final $f_{\text {res }}$ of $0.8 \mathrm{mHz}$ and a $T_{s}$ of $0.25 \mathrm{~s}$ are obtained.

Fig. 14 depicts the response of a cell battery. It can be observed that the converter noise becomes noticeable. Considering the impedance is measured at frequencies far from the switching harmonics, the response is filtered with a fifth order
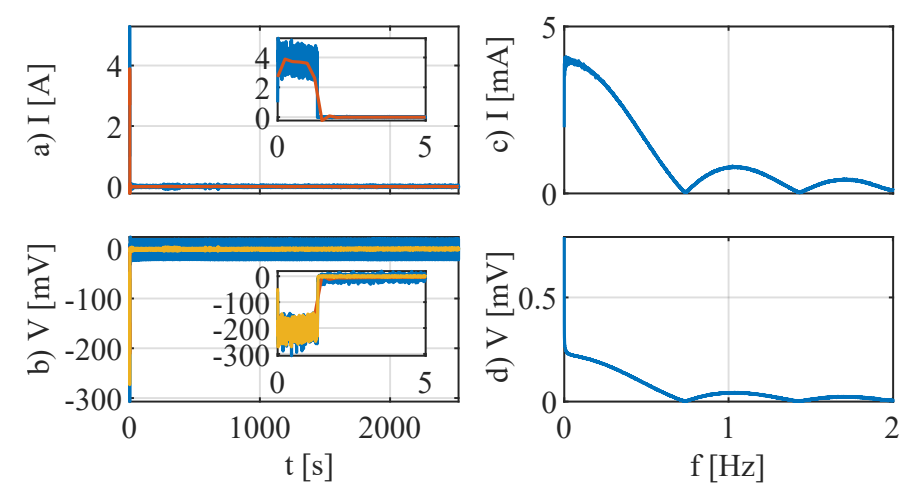

Fig. 14. Experimental results. VF response of the experimental test. a) Real (blue) and filtered(red) current. b) Real(blue), filtered(red)and estimated (yellow) cell voltage. c) DFT of the filtered current signal. d) DFT of the filtered voltage.

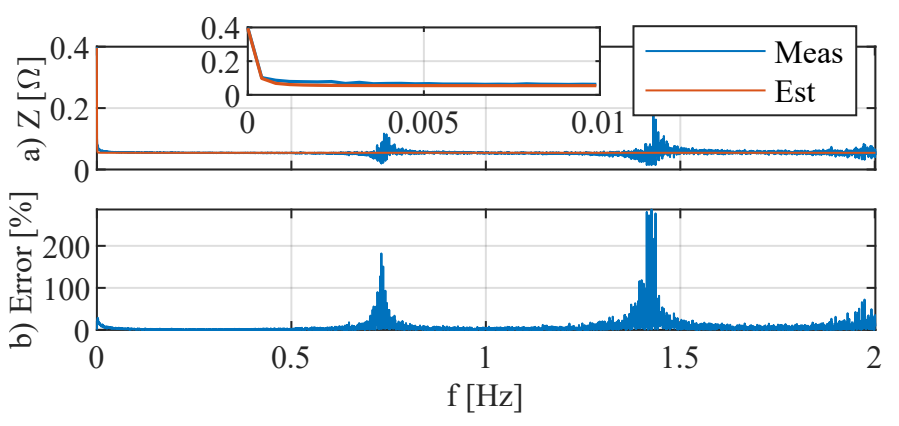

Fig. 15. Experimental results. a) Module in the frequency domain. b) Error between the experimental and estimated response.

low-pass-butterworth filter having a $0.1 \mathrm{~Hz}$ cutoff frequency, and the signal is resampled by a factor of $1 / 100$. The resulting impedance module in frequency domain is shown in Fig. 15a, both for the measured and the estimated parameters collected in Table IV. The mean error over the depicted range is below 1 $\%$, which means that the estimated parameters are valid. The error peaks that can be observed are due to the measurement noise at those frequencies close to the $1 / T_{p w}$ harmonics, as it can be observed in Fig. 15b.

It has to be remarked that for the experimental results, only the VF method has been implemented. This is becasue the used power source does not have current-control capability. That means, the excitation pulses are delivered as voltage pulses, being the resulting current distorted by the output filter. That makes the estimation too noisy and not comparable with the results obtained in simulations.

TABLE IV

EXPERIMENTAL RESULTS

\begin{tabular}{cccccc}
\hline & $R_{s}[\mathrm{~m} \Omega]$ & $R_{1}[\mathrm{~m} \Omega]$ & $C_{1}[\mathrm{~F}]$ & $R_{2}[\mathrm{~m} \Omega]$ & $C_{2}[\mathrm{~F}]$ \\
\hline $\mathrm{VF}$ & 46.8 & 0.008 & 341 & 1.3 & 5600 \\
\hline
\end{tabular}




\section{CONCLUSION}

The presented paper exposed a novel method that estimates the inner impedance of a battery and the inner states in real time using a current-pulse delivered by the converter. The parameters are initialized by means of a frequency method (VF), whereas the estimation in real time is performed by an RLS adaptive filter. Not only the method has been implemented but also some key input properties that enhance the final results have been remarked. Furthermore, the results are validated using a $3.2 A h$ module which consists of 826650 Lithium Iron Phosphate (LFP) cells in series.

\section{REFERENCES}

[1] M. A. Hannan, M. M. Hoque, A. Hussain, Y. Yusof, and P. J. Ker, "State-of-the-art and energy management system of lithium-ion batteries in electric vehicle applications: Issues and recommendations," IEEE Access, vol. 6, pp. 19362-19378, 2018.

[2] M. Nahvi and B. S. Hoyle, "Electrical impedance spectroscopy sensing for industrial processes," IEEE Sensors Journal, vol. 9, no. 12, pp. 18081816, Dec 2009.

[3] ISO 12405-4:2018 Electrically propelled road vehicles-Test specifcation for lithium-ion traction battery packs and systems - Part 4: Performance testing, ISO Std.

[4] D. Stroe, M. Swierczynski, A. Stroe, V. Knap, R. Teodorescu, and S. J. Andreasen, "Evaluation of different methods for measuring the impedance of lithium-ion batteries during ageing," in 2015 Tenth International Conference on Ecological Vehicles and Renewable Energies (EVER), March 2015, pp. 1-8.

[5] Z. Xia and J. A. A. Qahouq, "An online battery impedance spectrum measurement method with increased frequency resolution," in 2018 IEEE Applied Power Electronics Conference and Exposition (APEC), March 2018, pp. 1930-1933.

[6] S. Haykin and B. Widrow, Least Mean Square. John Wiley \& Sons, 2003.

[7] D. Simon, Optimal State Estimation. Wiley-Interscience, 2006.

[8] M. Hannan, M. Lipu, A. Hussain, and A. Mohamed, "A review of lithium-ion battery state of charge estimation and management system in electric vehicle applications: Challenges and recommendations," Renewable and Sustainable Energy Reviews, vol. 78, pp. 834 - 854, 2017. [Online]. Available: http://www.sciencedirect.com/science/article/ pii/S1364032117306275

[9] C. Zhang, W. Allafi, Q. Dinh, P. Ascencio, and J. Marco, "Online estimation of battery equivalent circuit model parameters and state of charge using decoupled least squares technique," Energy, vol. 142, pp. 678 - 688, 2018. [Online]. Available: http://www.sciencedirect.com/science/article/pii/S0360544217317127

[10] M. Oya, W. Sueki, Y. Hayakawa, K. Takaba, and M. Fukui, "Combined rls-kf method for soc and parameter estimation of lithium-ion batteries," Meeting Abstracts, vol. MA2017-02, no. 4, p. 260, 2017. [Online]. Available: http://ma.ecsdl.org/content/MA2017-02/4/260.abstract

[11] S. Herdjunanto, "Estimation of open circuit voltage and electrical parameters of a battery based on signal processed by recursive least square method using two separate forgetting factors," in 2016 6th International Annual Engineering Seminar (InAES), Aug 2016, pp. 67-71.

[12] B. Gustavsen and A. Semlyen, "Simulation of transmission line transients using vector fitting and modal decomposition," IEEE Transactions on Power Delivery, vol. 13, no. 2, pp. 605-614, April 1998.

[13] M. Ke, Y. Chiu, and C. Wu, "Battery modelling and soc estimation of a lifepo4 battery," in 2016 International Symposium on Computer, Consumer and Control (IS3C), July 2016, pp. 208-211.

[14] S. . Huang, B. . Huang, and F. . Pai, "An approach to measurements of electrical characteristics of lithium-ion battery with open-circuit voltage function," IET Power Electronics, vol. 5, no. 9, pp. 1968-1975, November 2012.

[15] G. F. Franklin, M. L. Workman, and D. Powell, Digital Control of Dynamic Systems, 3rd ed. Boston, MA, USA: Addison-Wesley Longman Publishing Co., Inc., 1997.
[16] H. He, R. Xiong, and J. Fan, "Evaluation of lithium-ion battery equivalent circuit models for state of charge estimation by an experimental approach," Energies, vol. 4, no. 4, pp. 582-598, 2011. [Online]. Available: http://www.mdpi.com/1996-1073/4/4/582

[17] B. Gustavsen and A. Semlyen, "Rational approximation of frequency domain responses by vector fitting," IEEE Transactions on Power Delivery, vol. 14, no. 3, pp. 1052-1061, July 1999.

[18] B. Gustavsen, "Improving the pole relocating properties of vector fitting," IEEE Transactions on Power Delivery, vol. 21, no. 3, pp. 15871592, July 2006.

[19] D. Deschrijver, M. Mrozowski, T. Dhaene, and D. D. Zutter, "Macromodeling of multiport systems using a fast implementation of the vector fitting method," IEEE Microwave and Wireless Components Letters, vol. 18, no. 6, pp. 383-385, June 2008.

[20] S. Haykin, Adaptive Filter Thoety, P. Hall, Ed., 1996. 